\title{
Revisiting Models for Spheroidal Graphite Growth
}

\author{
Mathias K. Bjerre ${ }^{1, a}$, Mohammed Azeem ${ }^{2, b}$, Peter D. Lee ${ }^{2, c}$, \\ Jesper H. Hattel ${ }^{1, d}$ and Niels S. Tiedje ${ }^{1, e, *}$ \\ ${ }^{1}$ Technical University of Denmark, Department of Mechanical Engineering, Produktionstorvet, \\ Building 425, $2800 \mathrm{Kgs}$. Lyngby, Denmark \\ ${ }^{2}$ School of Materials, The University of Manchester, Oxford Road, Manchester, M13 9PL, UK \\ amakabj@mek.dtu.dk, ${ }^{\mathrm{b}}$ mohammed.azeem@manchester.ac.uk, ${ }^{\mathrm{c}}$ peter.lee@manchester.ac.uk, \\ djhat@mek.dtu.dk, ensti@mek.dtu.dk
}

Keywords: Cast iron, Ductile iron, Modelling, Graphite, X-ray tomography

\begin{abstract}
Recent experiments resolved nucleation and growth of graphite during solidification of ductile cast iron in 3D and time using synchrotron X-ray tomography [1]. We use the experimental observations to analyse the relation between graphite growth rate and the state of the particle neighbourhood to pinpoint possible links between growth rate of individual graphite spheres and the overall solidification state. With this insight we revisit existing models for growth of spheroidal graphite and discuss possible modifications in order to describe the critical final stage of solidification correctly.
\end{abstract}

\section{Introduction}

Cast iron is a family of materials that has been in use for engineering purposes for thousands of years, and also today cast iron is essential to modern society. Engine components, brakes and hydraulics for trucks, cars, trains and ships are all made from cast irons. In modern wind turbines hubs, base frames and gears are made in cast iron.

The mechanical properties of cast irons are primarily determined by the shape, size and distribution of graphite crystals in a steel matrix. By varying the shape of graphite from large flakes to fine nodules we can change the cast irons properties from a material that is easy to cast and machine but brittle to a material that is ductile and has good strength and has excellent fatigue properties [2].

The effect of processing conditions on graphite shape size and distribution has been a topic for research for several decades. In recent years increasing focus has been on mathematical models for predicting microstructure and properties of cast irons.

A uni-nodular model that describes growth of graphite nodules and the austenite shell was proposed by Lesoult et al. [3]. It considers a spherically symmetric unit volume containing a single nodule surrounded by an austenite shell. The model assumes that the liquid melt surrounding the austenite shell acts as a reservoir for carbon from which the nodule can grow as carbon diffuses towards the nodule through the austenite shell. It is important to note that the model does not take into account the effect of the interaction of neighbouring unit volumes on the nodule growth rate neither by impingement of austenite shells nor by modified carbon concentrations in the liquid. Instead, the liquid surrounding the austenite shell effectively isolates solidifying spheres from one another.

The model was later built into a solidification model for thin walled ductile iron castings, where it was used to describe how cooling conditions and inoculation influence the nodule size distribution and formation of off-eutectic austenite [4]. However, compared with experimentally measured temperatures and nodule size distributions it has been shown that such a solidification model underestimates the nodule number density after solidification is complete. It has been speculated this is related to an inaccurate description of the nucleation and growth conditions at the end of solidification $[5,6]$.

It is the aim of the present paper to use results from recent experimental investigations into graphite growth and the resulting microstructure with the purpose of increasing the understanding of the factors influencing growth. 


\section{Experimental Details}

To gain insight into the relations between process conditions and microstructural evolution during solidification of ductile cast iron (DCI) an experiment was conducted on the I12 beam line at the Diamond Light Source synchrotron facility: A cylindrical sample of $2 \mathrm{~mm}$ in diameter and $8 \mathrm{~mm}$ in length was machined from a DCI casting with $3.6 \mathrm{wt} \% \mathrm{C}, 1.9 \mathrm{wt} \% \mathrm{Si}$ and $0.07 \mathrm{wt} \% \mathrm{Mg}$. To protect the sample from the surrounding atmosphere and to contain the sample during the experiment it was encapsulated in a quartz tube. Using an environmental cell described in detail in [7], the sample was heated to above the melting point and allowed to cool at a rate of $0.03{ }^{\circ} \mathrm{C} / \mathrm{s}$ while $3 \mathrm{D} \mathrm{X}$-ray tomograms were recorded. Details on imaging, construction of tomograms and filtering follow the methodology developed in [7-9] and are found in [1,10]. Binary images for quantification of particle shape and volume were obtained by filtering and segmenting reconstructed 3D volumes based on grey level variations representing differences in attenuation. It was not possible to separate liquid melt from austenite due to the limited attenuation difference between the two phases. A cross section of the sample with indication of the sub-volume analysed is shown in Fig. 1 where graphite nodules have formed.

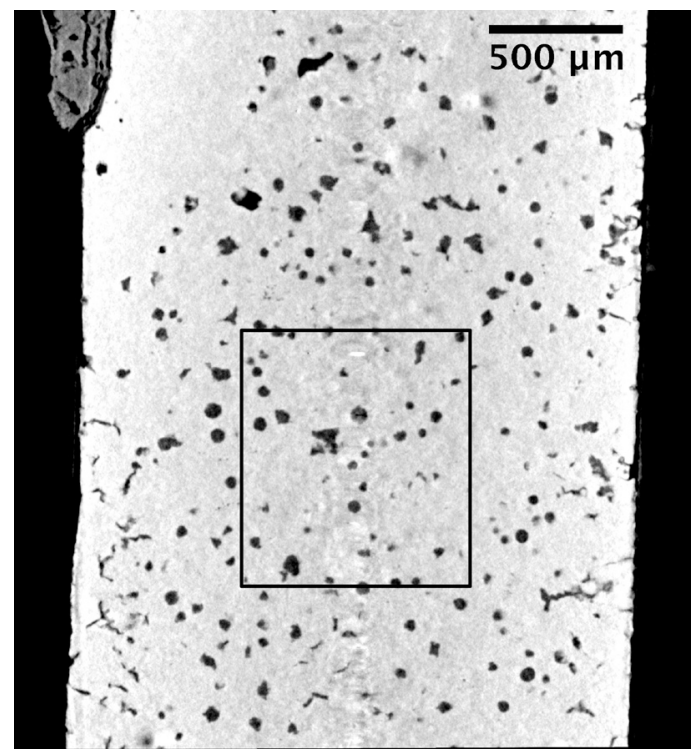

Figure 1. 2D cross section from tomogram obtained after solidification is complete. A sub-volume of $845 \times 845 \times 996 \mu \mathrm{m}$ (as indicated

by the rectangle) is used for the present analysis.

The sample solidified in the temperature range from $1138{ }^{\circ} \mathrm{C}$ to $1122{ }^{\circ} \mathrm{C}$ with compact graphite forming first at the sample periphery and spheroidal graphite forming subsequently in the central region. After solidification the sample was embedded in resin and polished with $3 \mu \mathrm{m}$ and $1 \mu \mathrm{m}$ diamonds in steps of approximately $20 \mu \mathrm{m}$ to reveal sequential layers through the sample. Each layer was investigated in Light Optical Microscope (LOM) and occasionally in Scanning Electron Microscope (SEM) to make element maps showing the spatial distribution of elements.

Nodules are tracked from one tomographic frame to the next. Each segmented volume is analysed using the $3 D$ Objects Counter in ImageJ. This way, each object in each tomographic frame is assigned a label unique to the analysed frame, and the particle volume, centroid coordinates and bounding box are obtained. Due to the very limited particle movement it is possible to track each individual nodule across time based on the position. This simple analysis was found to give good and consistent results overcoming issues of small particle movements and large changes in object volume between two time frames.

Nucleation of new graphite nodules cannot be directly observed due to the limited spatial resolution but also due to the fact that it takes $40 \mathrm{~s}$ to record a single tomogram. This way a new nodule can nucleate and grow to a detectable size within a single tomographic scan. Thus, the time at which a new nodule is observed does not correspond to the time of nucleation but is a good indication. The number of new nodules as a function of time and temperature is given by the open circles in Fig. 2a. No data is available at $t=440 \mathrm{~s}$ as the associated scan is corrupt. The figure shows that the nucleation rate is initially increasing but comes to a rapid decline as solidification is nearly 
complete at $t=480 \mathrm{~s}$. The initiation of nodule formation marks the onset of the eutectic solidification.

\section{Spheroidal Graphite Growth}

Focusing now on the growth of spheroidal Fig. 2a also presents the relation between the time at which a nodule is first observed and its average final nodule volume after solidification. The figure clearly shows that the general trend is that the more time a nodule has available to grow the larger it becomes. This is in good agreement with the understanding expressed e.g. the model by Lesoult et al. [3]. Thus, when observing a room temperature microstructure it is likely that large nodules have nucleated before smaller nodules.

Focussing now on individual nodules Fig. $2 \mathrm{~b}$ shows the volume as a function of time for five particles distributed throughout the considered sub-volume. The shape of each graphite nodule is quantified by calculating the sphericity which is defined as $\Psi=\left(36 \pi V^{2}\right)^{1 / 3} A^{-1}$ where $V$ is the object volume and $A$ its surface area.

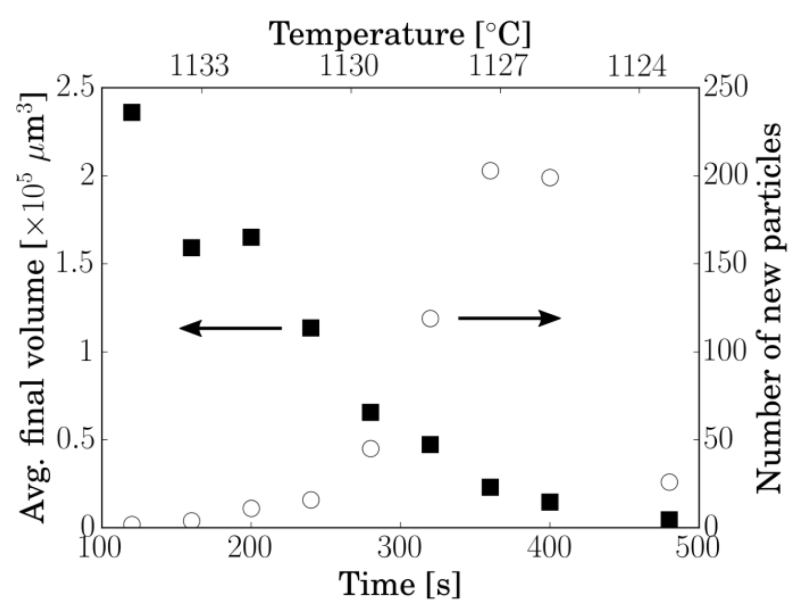

$\mathbf{a}$

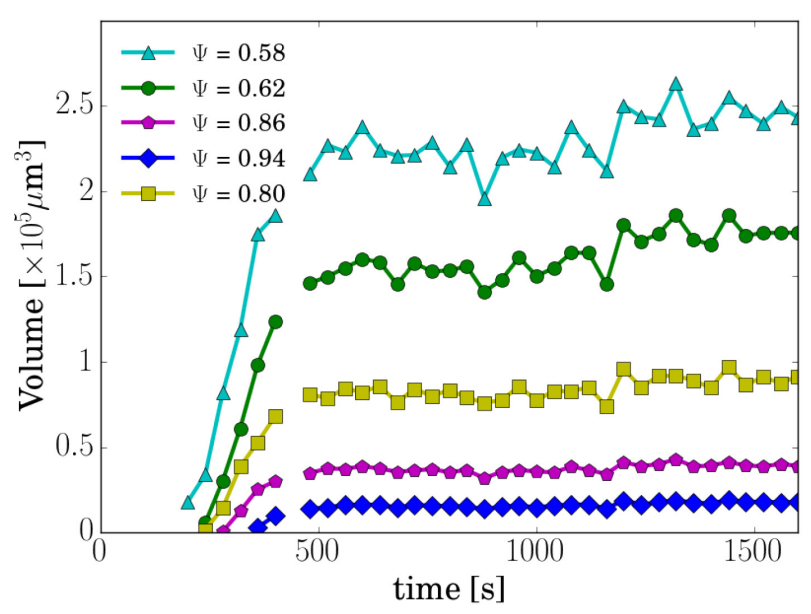

b

Figure 2. a: The average final nodule volume plotted as a function of time and temperature at first observation (black squares, left axis) as well as the number of new graphite particles observed at each instance of time (open circles, right axis). b: Volume of 5 graphite nodules as a function of time. Note that the scan at $440 \mathrm{~s}$ was corrupt resulting in interrupted curves.
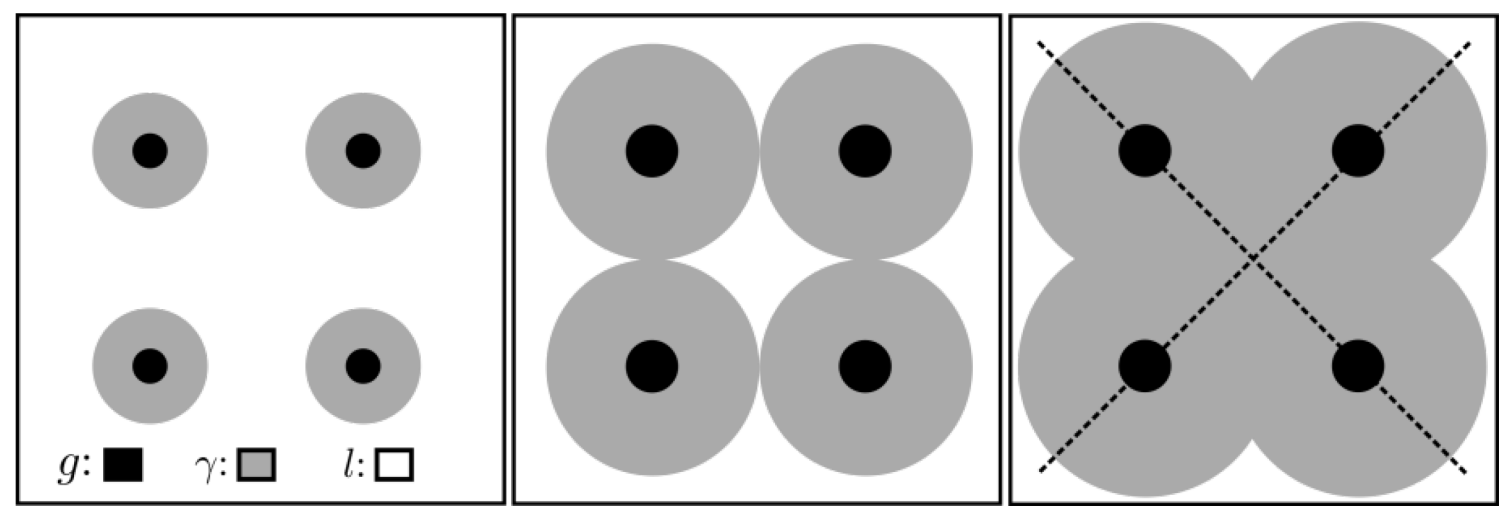

Figure 3. Coalescence of solidifying spheres at three stages during growth of encapsulated nodules. Graphite is labelled $g$, austenite $\gamma$ and liquid $l$. Symmetry lines are indicated by the dashed lines which are used in Fig. 4 to show carbon concentration variations.

$\Psi=1$ means that the object is perfectly spherical and less spherical objects have $\Psi<1$. The particle sphericities after solidification are indicated in the figure legend and show that the curves represent the growth of particles of a range of shapes. Each particle displays a growth stage up until $480 \mathrm{~s}$ after which the particle volume is constant within the measurement uncertainty. The 
experimental data indicates that the volume of the graphite particles grow monotonically with time. The collective transition to a plateau of constant particle volume indicates that graphite precipitation ends at approximately the same time. The figure also illustrates the correspondence between the time of first observation and the final volume of the particle observed in Fig. 2a. If we compare the particles with $\Psi=0.62$ and 0.80 (green circles and yellow squares respectively) which start out with similar sizes at the same time instance highlights that considerable individual variations in growth rate exist. As discussed by [1] irregular nodules $(\Psi<0.75)$ tend to grow faster than their regular counterparts. However, variations in growth rate exist even between nodules which are otherwise comparable as discussed in the following section.

\section{Discussion}

Graphite growth is driven by the flux of carbon which is the result of a concentration gradient in the surrounding matrix. The uni-nodular model assumes that the concentration gradients, which to a large extent are maintained due to the presence of the carbon-rich liquid, remain large throughout solidification. Thus, nodules are considered unaffected by the presence of their neighbours. However, considering the simple case of four nodules and their austenite shells during solidification, Fig. 3 illustrates that this assumption probably is a poor approximation at late stages of solidification. Figure 4 shows a schematic drawing of the concentration profile along the symmetry lines in Fig. 3. The figure illustrates that the impingement of austenite shells results in overlapping diffusion fields and probably an anisotropic concentration profile which cannot be described by the assumed quasi-steady state concentration profile. Especially, the region between nodules is likely depleted in carbon. Further, since austenite dendrites are often present during solidification of DCI, the idealised arrangement of austenite and graphite within the uni-nodular model is not necessarily a correct. Instead multiple nodules can be embedded in the same austenite grain.

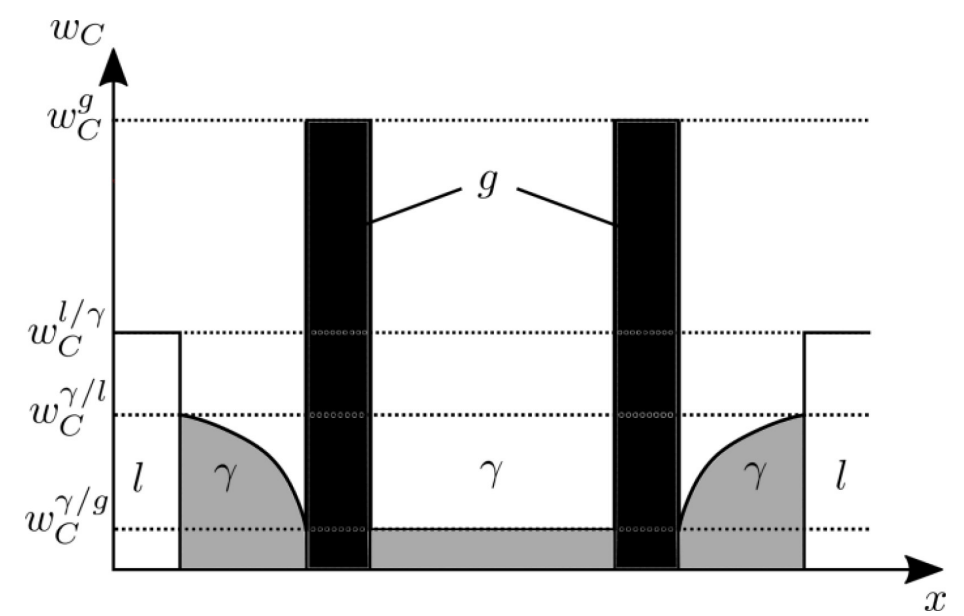

Figure 4. Schematic drawing of the carbon concentration profile along one of the symmetry lines in Fig. 3. 

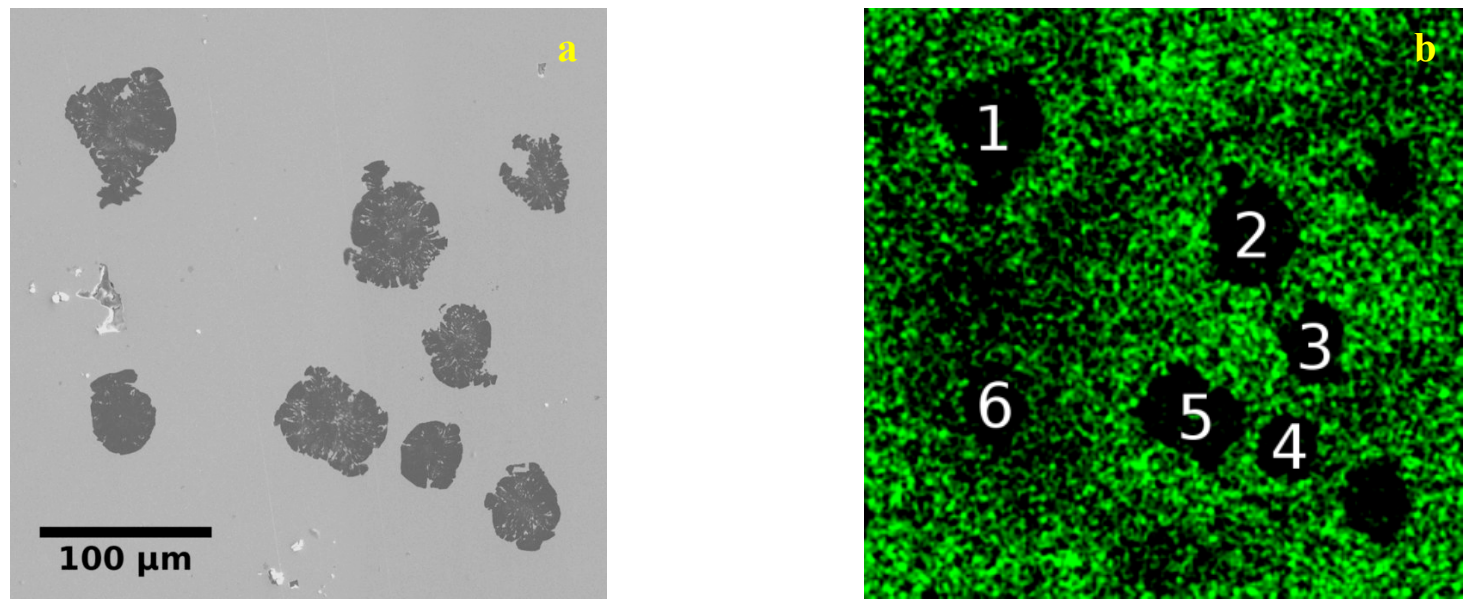

Figure 5. a: Electron microscope image of the microstructure. b: Si element map of the section showed in figure 5a obtained by EDS. Numbers refer to the particles described in Fig. 6.

Figure 5a presents an example of the microstructure of the considered sample obtained by SEM after solidification. The distribution of Silicon in the same area obtained by Energy-dispersive X-ray spectroscopy (EDS) is shown in Fig. 5b. Since Silicon segregates negatively during solidification low-Si areas, which appear dark in Fig. 5b, indicate where the sample solidified last. Thus, according to Fig. $5 \mathrm{~b}$ the nodule with the label 6 formed in the last-to-solidify melt. The volume of the nodules 1-6 as function of time obtained from the in situ observations are presented in Fig. 6. The plot shows that among these six nodules, nodule 2 and 5 formed first and nodule 6 formed last, probably during the missing scan at $\mathrm{t}=440 \mathrm{~s}$. The latter confirms the indication from the Si-map of Fig. 5b. The nodules labelled 2 and 5 are not the largest particles after solidification is complete although they nucleated first. Instead they are exceeded in volume by nodule 1 which forms one time instance later. It is also interesting to note that nodule 3 and 4 formed around the same time as nodule 1 but grow at a lower rate resulting in a much lower final volume. The final volume of nodule 6 is comparable to that of nodule 3 and 4 although it forms very late in the solidification process. These observations show that notable exceptions to the general trend

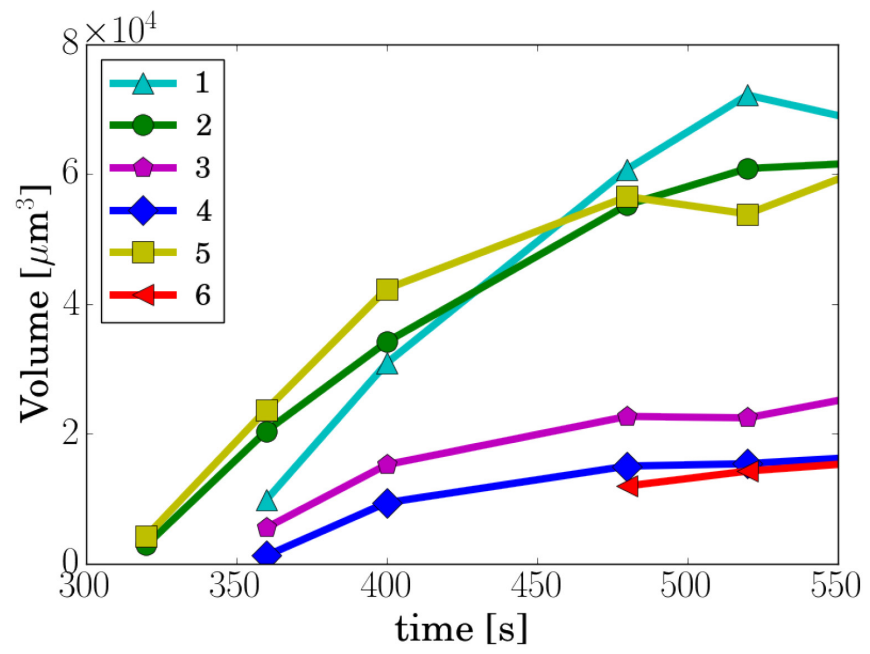

Figure 6. Measured volume as function of time for the particles labelled 1-6 in Fig. 5b.

expressed by Fig. 2a exist and most likely are linked to the very local solidification conditions.

One may ask: Which differences in the growth conditions are accountable for the variations in growth rate observed between nodule 1 and 3? Figure 5b suggests that nodule 1 and 6 formed in a volume with high fraction liquid while particles 3 and 4 are in a location where the distance to the nearest neighbours is short. With the discussion of Fig. 3 and 4 in mind there seems to be two possible explanations: 
1. A high local density of nodules might lead to low growth rates because of competition for carbon. A graphite particle nucleating in the liquid centre between the four encapsulated nodules of the second frame of Fig. 3 will relatively quickly be surrounded by an austenite matrix with small carbon concentration gradients. This will limit the growth rate of the new nodule.

2. The carbon-rich liquid plays an important role in maintaining the local carbon concentration gradients. Thus, if a nodule is located next to the liquid it is likely that it will grow faster than a nodule which is located far from the liquid.

The effect of particle position with respect to the liquid can only be investigated through postmortem mappings of the Silicon segregation on 2D sections since the solid-liquid interface is not available from the synchrotron $\mathrm{CT}$ data in the presented experiment. This limits the extent of the above analysis. A possible solution is to reconstruct the microstructure in $3 \mathrm{D}$ using the microscope images obtained during serial sectioning but this strategy was not pursued in the present investigation.

The presented analysis has shown the importance of the local conditions for the growth rate of graphite nodules during solidification. It is clear that the idealised spherical symmetry often assumed by models does not necessarily provide a correct basis for predicting the nodule growth rate. Significant variations in growth rate exist between similar neighbouring particles indicating that local conditions can vary significantly over small distances. In spite of this the uni-nodular model provides relatively good predictions of the nodule growth rate as recently shown [1].

Previous investigations have suggested modifications of the nodule growth model taking into account the effect of impinging austenite shells on graphite growth. Su et al. [11] suggested that austenite growth is limited by impingement above $f_{s}=0.5$ where $\mathrm{f}_{\mathrm{s}}$ is the solid fraction but did not apply the modification to graphite growth. Boeri [12] took inspiration from this approach and assumed that the nodule growth rate decreased with increasing fraction solid by multiplying the growth rate by $0.9 \cdot\left(1-f_{s}\right)^{2 / 3}$. Modifications along these lines might be a viable way to take into account the some of the observations made in the present investigation. Current models for nodule growth have suffered from limited possibilities for direct validation through comparison to measurements on single nodule growth. This data is now available [1].

The Cellular Automata (CA) model proposed by Zhu et al. [13] seems to provide a possibility to evaluate the effects of carbon concentration fields which are not spherically symmetric. They find a large effect of competition for carbon among neighbouring nodules growing freely in the melt supporting the above suggestion that competition is relevant among encapsulated nodules. While the model is considered computationally efficient by the authors it does not seem likely that this type of model can be applied in casting scale simulations. Instead, the CA model may be a good tool for evaluating the effect of competition and impingement of austenite shells on the nodule growth rate. Such insights can be incorporated into simplified 1D models which are necessary when predicting the development of a large population of nodules. Kampmann-Wagner numerical (KWN) models are frequently applied to describe the kinetics of size distributions of spherical precipitates in solid state [14,15]. Such models are more computationally efficient than the related approach by Lacaze et al. [16]. Applying simplified models in a KWN model for nodule growth during solidification and solid state growth would provide a computationally "cheap" framework for predicting nodule size distributions in industrial castings. Such results would be a very useful basis for estimating the local mechanical properties of a cast component.

\section{Conclusion}

Observations on spheroidal graphite growth from in situ observations obtained by synchrotron $\mathrm{X}$-ray tomography have been presented. The data shows a clear relation between early nodule formation and large final nodule volume and vice versa. However, the experimental observations also showed a significant effect of the local conditions in terms of distance to neighbours and especially the distance to the carbon-rich liquid on the graphite nodule growth rate during solidification. It is clear that the spherical symmetry assumed by the uni-nodular model is not a 
good approximation to the actual arrangement of austenite and graphite at late stages of solidification. This should be taken into account by future models for nodule growth during solidification.

\section{References}

[1] M. Azeem, M. Bjerre, R. Atwood, N. Tiedje, P. Lee, Revealing graphite's multitude of growth modes in a metal-carbon alloy, Submitted. (2017).

[2] N.S. Tiedje, Solidification, processing and properties of ductile cast iron, Mater. Sci. Technol. 26 (2010) 505-514. doi:10.1179/026708310X12668415533649.

[3] G. Lesoult, M. Castro, J. Lacaze, Solidification of spheroidal graphite cast iron - I. Physcial modelling, Acta Metall. 46 (1998) 983-995.

[4] K.M. Pedersen, J.H. Hattel, N. Tiedje, Numerical modelling of thin-walled hypereutectic ductile cast iron parts, Acta Mater. 54 (2006) 5103-5114.

[5] K.M. Pedersen, N.S. Tiedje, Undercooling and nodule count in thin walled ductile iron castings, Int. J. Cast Met. Res. 20 (2007) 145-150. doi:10.1179/136404607X239816.

[6] M. Bjerre, N. Tiedje, J. Thorborg, J. Hattel, Modelling the solidification of ductile cast iron parts with varying wall thicknesses, IOP Conf. Ser. Mater. Sci. Eng. 84 (2015) 12038.

[7] M.A. Azeem, P.D. Lee, A.B. Phillion, S. Karagadde, P. Rockett, R.C. Atwood, L. Courtois, K.M. Rahman, D. Dye, Revealing dendritic pattern formation in $\mathrm{Ni}$, Fe and Co alloys using synchrotron tomography, Acta Mater. (2017).

[8] S. Karagadde, P.D. Lee, B. Cai, J.L. Fife, M.A. Azeem, K.M. Kareh, C. Puncreobutr, D. Tsivoulas, T. Connolley, R.C. Atwood, Transgranular liquation cracking of grains in the semi-solid state., Nat. Commun. 6 (2015) 8300. doi:10.1038/ncomms9300.

[9] K.M. Kareh, P.D. Lee, R.C. Atwood, T. Connolley, C.M. Gourlay, Revealing the micromechanisms behind semi-solid metal deformation with time-resolved X-ray tomography, Nat. Commun. 5 (2014) 4464. doi:10.1038/ncomms5464.

[10] M. Bjerre, In situ observations of graphite formation during solidification of cast iron, Ph.D.-thesis, Technical University of Denmark, 2017.

[11] K.C. Su, I. Ohnaka, I. Yamauchi, T. Fukusako, Computer Simulation of Solidification of Nodular Cast Iron, in: H. Fredriksson, M. Hillert (Eds.), Proc Int Symp Met. Cast Iron, 3rd, Stockholm, 1985: pp. 181-190.

[12] R.E. Boeri, The solidification of ductile cast iron, The University of British Columbia, 1989.

[13] M. Zhu, L. Zhang, H. Zhao, D.M. Stefanescu, Modeling of microstructural evolution during divorced eutectic solidification of spheroidal graphite irons, Acta Mater. 84 (2015) 413-425. doi:10.1016/j.actamat.2014.10.057.

[14] O.R. Myhr, Ø. Grong, Modelling of non-isothermal transformations in alloys containing a particle distribution, Acta Mater. 48 (2000) 1605-1615. doi:10.1016/S1359-6454(99)00435-8.

[15] J.D. Robson, P.B. Prangnell, Dispersoid precipitation and process modelling in zirconium containing commercial aluminum alloys, Acta Mater. 49 (2001) 599-613. doi:10.1016/S13596454(00)00351-7.

[16] J. Lacaze, M. Castro, G. Lesoult, Solidification of spheroidal graphite cast irons - II. Numerical simulation, Acta Mater. 46 (1998) 997-1010. 